Editorial

\title{
Noncoding RNAs: the fourth dimension of the human genome
}

\author{
Abbreviations: lncRNA, long non-coding RNA; miR, micro \\ RNA; lincRNAs, long intergenic RNA; SNP, single nucleotide \\ polymorphism
}

\section{Editorial}

The human genome encoding the three billion letters of DNA alphabet continues to deliver surprises and raise new questions. ${ }^{1,2}$ The initial focus of the genome project was on the discovery of gene targets for diagnosis and therapy. ${ }^{3}$ Until recently, the knowledge base of the genome largely revolved around three dimensions: DNA, RNA and protein. A vast amount of the human genome once considered Dark Matter or junk DNA has become an area of intense research. ${ }^{4-9}$ It is estimated that over $93 \%$ of the human genome is transcribed. ${ }^{10}$ The role of this huge amount of the transcriptome in biology is however, unclear. This part of the genome, the noncoding RNAs (ncRNAs) can be rightly be considered the fourth dimension of the genome.

The ncRNAs comprise the long non-coding RNAs (lncRNAs), the long intergenic RNAs (lincRNAs), endogenous antisense RNAs, the microRNAs (miRs) and pseudogenes-derived lncRNAs. ${ }^{7}$ The current version of the GENCODE database (v 22) estimates there to be 25,794 ncRNAs in humans. ${ }^{11,12}$ The ncRNAs are crucial in gene regulation exerting their regulatory role at the chromatin, transcriptome and proteome level. ${ }^{13}$ The regulation of the target genes by the ncRNAs occurs at the level of RNA and protein stabilization, protein binding and protein translocation or processing. ${ }^{9}$

Originally thought to be non-protein coding transcripts, at least some of the ncRNAs now seem to be capable of coding for Open Reading Frames. ${ }^{14,15}$ Thus, eventually the terminology non-coding will have to evolve to accurately reflect the differences between the coding and the non-coding nature of the transcriptome. Increasing evidence in the ncRNA field of research is beginning to challenge our current understanding of the human genome. Pseudogene-derived lncRNAs are emerging as key regulators of gene expression and as a reservoir for miRs and endogenous antisense transcripts. ${ }^{16}$ In addition, various Genome Wide Association Studies (GWAS) have identified a vast majority of disease-associated Single Nucleotide Polymorphisms (SNPs) which are present at the non-coding intronic or intergenic regions of the genes. ${ }^{17,18}$ These regions were neglected in the past because of the focus on the protein coding regions. However, the attention is beginning to shift to this unexplored region of the human genome. Understanding the impact of these SNPs is going to allow us to develop a better understanding of diverse disease-associated phenotypes in the future.

The ncRNAs including the miRs are of major interest for novel therapeutics as well as diagnostic biomarker development. ${ }^{4,19,20}$ Numerous tools are becoming available to study the ncRNAs. ${ }^{21-26}$ A microRNAs miR 222, is being developed as a therapeutic for hepatocellular carcinoma. $^{27}$ The Federal Drug Agency (FDA) approved its first lncRNA, the Prostate cancer antigen 3 (PCA3) as a urinary biomarker for prostate cancer diagnosis. ${ }^{28}$ The miRs because of the stability in body fluids due to the secretome nature involving the
Volume 2 Issue 2 - 2015

\author{
Ramaswamy Narayanan \\ Department of Biological Sciences, Charles E. Schmidt College \\ of Science, Florida Atlantic University, USA
}

\begin{abstract}
Correspondence: Ramaswamy Narayanan, Department of Biological Sciences, Charles E. Schmidt College of Science, Florida Atlantic University, Boca Raton, FL3343I, USA, Tel 5612972247, Fax 5612973859, Email rnarayan@fau.edu
\end{abstract}

Received: March 21, 2015 | Published: March 28, 2015

exosomes, offer tremendous opportunities for biomarker potential for diagnosis and prognosis for cancer and other diseases. ${ }^{19,29-31}$

The ncRNAs are the next frontier in molecular biology. The fruits of research in the ncRNA arena in the next decade are likely to challenge the current dogma in molecular biology. As we begin to understand more about the role of the entire transcriptome in gene regulation, our understanding of biology and function of a gene will achieve greater clarity. This is likely to lead into novel therapy diagnosis for diverse diseases.

\section{Acknowledgements}

I thank Jeanine Narayanan for editorial assistance. This work was supported in part by the Genomics of Cancer Fund, Florida Atlantic University Foundation.

\section{Conflict of interest}

The author declares no conflict of interest.

\section{References}

1. Lander ES, Linton LM, Birren B, et al. Initial sequencing and analysis of the human genome. Nature. 2001;409(6822):860-921.

2. Venter JC, Adams MD, Myers EW, et al. The sequence of the human genome. Science. 2001;291(5507):1304-1351.

3. Hopkins AL, Groom CR. The druggable genome. Nat Rev Drug Discov. 2002;1(9):727-730.

4. Martin L, Chang HY. Uncovering the role of genomic "dark matter" in human disease. J Clin Invest. 2012;122(5):1589-1595.

5. Hauptman N, Glavac D. MicroRNAs and long non-coding RNAs: prospects in diagnostics and therapy of cancer. Radiol Oncol. 2013;47(4):311-318.

6. Huang Y, Regazzi R, Cho W Editorial on "Emerging roles on long noncoding RNAs in neurological diseases and metabolic disorders" Out of Darkness - Long Noncoding RNAs Come of Age. Frontiers in Genetics. 2014:5.

7. Wright MW. A short guide to long non-coding RNA gene nomenclature. Hum Genomics. 2014;8:7. 
8. Yang G, Lu X, Yuan L. LncRNA: a link between RNA and cancer. Biochim Biophys Acta. 2014;1839(11):1097-1109.

9. Iyer MK, Niknafs YS, Malik R, et al. The landscape of long noncoding RNAs in the human transcriptome. Nat Genet. 2015;47:199-208.

10. Consortium EP, Birney E, Stamatoyannopoulos JA, et al. Identification and analysis of functional elements in $1 \%$ of the human genome by the ENCODE pilot project. Nature. 2007;447(7146):799-816.

11. Harrow J, Frankish A, Gonzalez JM, et al. GENCODE: the reference human genome annotation for The ENCODE Project. Genome Res. 2012;22(9):1760-1774.

12. Cunningham F, Amode MR, Barrell D, et al. Ensembl 2015. Nucleic Acids Res. 2015;43(Database issue):D662-D669.

13. Yang G, Lu X, Yuan L. LncRNA: A link between RNA and cancer Biochimica et Biophysica Acta (BBA) - Gene Regulatory Mechanisms. 2014;1839(11):1097-1109.

14. Delgado A, Chapado MJ, Brandao P, et al. Atlas of the Open reading Frames in human diseases: Dark matter of the human genome. MOJ Proteomics Bioinform. 2015;2(1):00036.

15. Kageyama Y, Kondo T, Hashimoto $\mathrm{Y}$. Coding vs non-coding: translatability of short ORFs found in putative non-coding transcripts. Biochimie. 2011;93(11):1981-1986.

16. Milligan MJ, Lipovich L. Pseudogene-Derived LncRNAs: Emerging Regulators of Gene Expression. Front Genet. 2015;5:476.

17. Hindorff LA, Sethupathy P, Junkins HA, et al. Potential etiologic and functional implications of genome-wide association loci for human diseases and traits. Proc Natl Acad Sci U S A. 2009;106(23):9362-9367.

18. Kellis M, Wold B, Snyder MP, et al. Defining functional DNA elements in the human genome. Proc Natl Acad Sci U S A. 2014;111(17):6131-6138.

19. Shen J, Stass SA, Jiang F. MicroRNAs as potential biomarkers in human solid tumors. Cancer lett. 2013;329(2):125-136.

20. Zarate R, Boni V, Bandres E, et al. MiRNAs and LincRNAs: Could they be considered as biomarkers in colorectal cancer? Int J Mol Sci. 2012;13(1):840-865.
21. Xiao F, Zuo Z, Cai G, et al, miRecords: an integrated resource for microRNA-target interactions. Nucleic Acids Res. 2009;37(Database issue):D105-D110.

22. Xie B, Ding Q, Han H, et al. miRCancer: a microRNA-cancer association database constructed by text mining on literature. Bioinformatics. 2013;29(5):638-644

23. Jiang Q, Wang Y, Hao Y, et al. miR2Disease: a manually curated database for microRNA deregulation in human disease. Nucleic Acids Res. 2009;37(Database issue):D98-D104

24. Mortazavi A, Williams BA, McCue K, et al. Mapping and quantifying mammalian transcriptomes by RNA-Seq. Nat Methods. 2008;5(7):621628

25. Fritah S, Niclou SP, Azuaje F. Databases for lncRNAs: a comparative evaluation of emerging tools. RNA. 2014;20(11):1655-1665.

26. Guffanti A, Simchovitz A, Soreq H. Emerging bioinformatics approaches for analysis of NGS-derived coding and non-coding RNAs in neurodegenerative diseases. Front Cell Neurosci. 2014;8:89.

27. Garofalo M, Quintavalle C, Romano G, et al. miR221/222 in cancer: their role in tumor progression and response to therapy. Curr Mol Med. 2012;12(1):27-33.

28. Crawford ED, Rove KO, Trabulsi EJ, et al. Diagnostic performance of PCA3 to detect prostate cancer in men with increased prostate specific antigen:a prospective study of 1,962 cases. J Urol. 2012;188(5):17261731.

29. Candelario KM, Steindler D. The role of extracellular vesicles in the progression of neurodegenerative disease and cancer. Trends Mol Med. $2014 ; 20(7): 368-374$

30. Lujambio A, Lowe SW. The microcosmos of cancer. Nature 2012;482(7385):347-355.

31. Costa PM, Pedroso de Lima MC. MicroRNAs as Molecular Targets for Cancer Therapy: On the Modulation of MicroRNA Expression. Pharmaceuticals. 2013;6(10):1195-1220. 